\title{
Neonatal Crohn's disease with Oral ulcer as the first symptom caused by a compound heterozygote mutation in IL-10RA: a case report
}

Hongyan Lv ${ }^{1,2^{*}}$, Baojun Qiao ${ }^{1}$, Liyuan Fang ${ }^{1}$, Lihong Yang ${ }^{1}$, Qiuli Wang ${ }^{1}$, Sujing Wu' ${ }^{1}$, Pengshun Ren ${ }^{1}$ and Lianxiang $\mathrm{Li}^{2,3}$

\begin{abstract}
Objective: To investigate the clinical and genetic characteristics of neonatal Crohn's disease (CD), improve recognition of neonatal $C D$, and reduce the number of patients that are missed or misdiagnosed.

Methods: A 10-day-old Chinese girl with oral ulcers was admitted to the Department of Neonatology. She later developed a rash and perianal disease, but without diarrhea and stool abnormalities. The patient and her parents underwent next-generation sequencing.

Results: The results showed that the patient carries a compound heterozygous mutation in the interleukin-10 receptor A (IL-10RA) (NM_001558.3) gene. One heterozygous mutation was c.301 c> T, P. (Arg 101 Trp) in exon 3 of IL-10RA (a missense mutation), and the other was C. 537G > A, P. (Thr $179=$ ) in exon 4 of IL 10RA (a synonymous mutation). The patient's father also carries the c.301 c > T, P. (Arg 101 Trp) heterozygous mutation in exon 3 of IL10RA, whereas her mother carries the c.537G > A, P. (Thr 179 =) heterozygous mutation in exon 4 of IL-10RA.
\end{abstract}

Conclusions: The results show that a compound heterozygous mutation in IL-10RA is associated with neonatal CD. Oral ulcers with a rash and perianal disease may be an early symptom of neonatal CD; therefore, such patients should undergo genetic identification as soon as possible.

Keywords: Neonate, Oral ulcer, Crohn's disease, Inflammatory bowel disease, Interleukin-10 receptor a, Genetic mutation

\section{Introduction}

Crohn's disease (CD) is a subtype of inflammatory bowel disease (IBD); it is a chronic idiopathic inflammatory disorder of the gastrointestinal tract with unknown etiology. In $\mathrm{CD}$, the inflammation is often transmural and may involve the whole gastrointestinal tract. Clinical manifestations include abdominal pain, diarrhea, intestinal granulomas, strictures, fistulas, perforation, fever,

\footnotetext{
* Correspondence: 3581479478@qq.com

'Department of Neonatology, Handan Maternal and Child Health Care Hospital, No. 6, Li Ming Street, Peace Road, Handan City 056001, Hebei Province, China

${ }^{2}$ Department of Neonatal Pathology, Handan Maternal and Child Health Care Hospital, No. 6, Li Ming Street, Peace Road, Handan City 056001, Hebei Province, China

Full list of author information is available at the end of the article
}

and malnutrition. The main pathological changes that occur during $\mathrm{CD}$ are thickening of the intestinal wall, non-cheese like granuloma nodules, and ulcers of varying depths on the mucosal surface. Depending on ethnicity, region, and age, the incidence of $\mathrm{CD}$ differs; the incidence rate in Europe is significantly higher than that in Asia. According to previous reports, the incidence of CD was found to be $0.848-2.7$ per 100,000 [1-3]. CD often occurs in children and young adults, and the incidence rate is reported to be slightly higher in men than in women.

Neonatal CD is very rare. In the present study, we report the case of a 10-day-old girl with neonatal CD. The first symptom was oral ulcers. The patient later developed a rash, fever, perianal masses, and perianal 
pyoderma, without any clinical symptoms such as diarrhea, mucous stools, or bloody stools. As a result, we suspected an autoimmune disease; therefore, the patient and her parents underwent genetic sequencing. Finally, she was diagnosed with neonatal CD.

\section{Materials and methods}

\section{Case presentation}

A 10-day-old girl (Han Chinese) with oral ulcers was admitted to the Department of Neonatology in our hospital on July 22, 2018. After treatment, her condition did not improve; therefore, we suspected an autoimmune disease. This study was conducted in accordance with the Declaration of Helsinki and was approved by the Medical Ethics Committee of Handan Maternal and Child Health Care Hospital of Hebei Province. Written informed consent was provided by patient's parents.

Upon admission, the patient's oral cavity was found to have many ulcers 10 days after birth. In addition, the external auditory canal had abnormal secretions. The shape of the oral ulcers were irregular, as was the size of the ulcers, and the surface was covered with a layer of white plaque that was not easy to wipe away (Fig. 1). However, the patient had no clinical manifestations of diarrhea, vomiting, or bloody stools. Upon a physical examination, the patient was conscious and did not appear malnourished, had a body temperature $36.9^{\circ} \mathrm{C}$, heart rate of 136 beats minute, respiratory rate of 36 breaths per minute, blood pressure of $85 / 50 \mathrm{mmHg}$, and weighed $4.9 \mathrm{~kg}$. There were three ulcers in the mucosa of the upper palate and the uvula had three ulcers, with varying shapes and sizes. The patient's heart, lungs, and peristaltic waves were all normal. A neurological examination showed that the patient had no focal neurological impairments. After admission, she was initially diagnosed with neonatal oral ulcers and neonatal purulent otitis media. She received oral care, ofloxacin ear drops, amoxicillin, clavulanate potassium, antibiotics, and human immunoglobulin. On the fourth day after admission, she developed a fever with a red rash on her face, which was rice-like in appearance and pustule. The rash spread from her face to her armpits, chest area, groin, and perianal area. The laboratory results are shown in Table 1. The Erythra test was fungus positive and the fecal occult blood test was weakly positive. There were no abnormalities in coagulation factors. Therefore, she was treated with calamine lotion and fusidic acid cream, and with supportive care, the patient's condition became slightly alleviated. However, 31 days after admission, she developed fever, oral ulcers, perianal pyoderma and perianal mass, and moderate anemia $(\mathrm{Hb}$ : $72 \mathrm{~g} / \mathrm{L}$ ) (Fig. 2). The patient continued to be treated with calamine lotion, fusidic acid cream, antibiotics, and supportive care, and as a result, her symptoms were temporarily improved.

When taking a family history, we found that the patient' brother developed fever, diarrhea, oral ulcer, and hyperbilirubinemia 5 days after birth, which developed in to septicemia and intestinal obstruction. Unfortunately, the patient' brother died 30 days after birth and so was unable to provide corresponding genetic test results. According to the patient's family history, we considered that the patient had an autoimmune disease and suggested that the patient and her parents should carry out genetic testing in order to obtain a definitive diagnosis.

\section{Next generation sequencing (NGS)}

Genomic DNA was extracted from the patient's and the patient's parents peripheral blood. DNA was extracted from the blood sample using the QIAamp DNA extraction kit (QIAGEN NV, Hilden, the Netherlands), per the manufacturer's instructions. The extracted DNA was purified using magnetic beads after DNA enzyme fragmentation. Next, the sample was PCR amplified and connected to the upper joint sequence. After capture and purification by TruSight One Sequencing Panel (Illumina Inc., USA) twice, the final library was obtained by PCR amplification and purification once again. The exonic regions of 4811 genes were sequenced by NextSeq500 Sequencing apparatus (Illumina Inc., USA). All

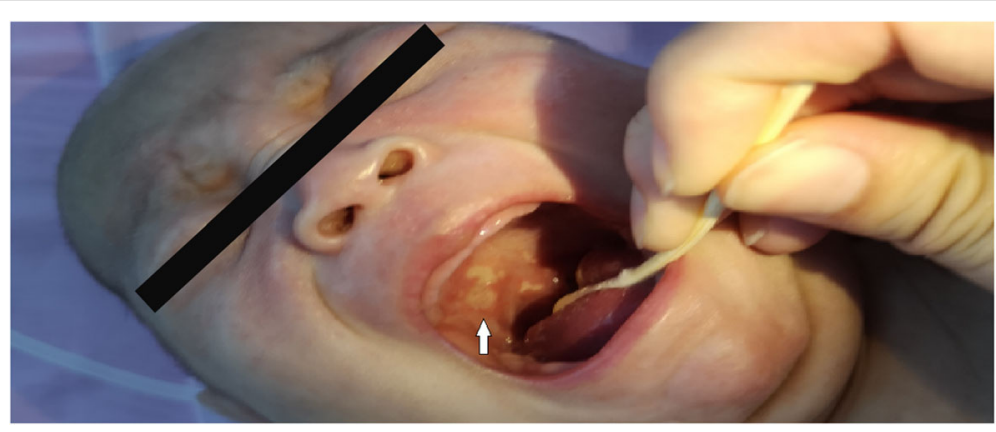

Fig. 1 The patient had three ulcers on the mucosa of the upper palate and uvula. The surface of the ulcers is covered with a layer of white plaque (arrow) 
Table 1 Laboratory parameters

\begin{tabular}{|c|c|c|}
\hline Laboratory index & Outcome & $\begin{array}{l}\text { Reference } \\
\text { value }\end{array}$ \\
\hline Red blood cell count (RBC) & $\begin{array}{l}47 \times 10^{-12} / \\
L\end{array}$ & $4-5.2 \times 10^{-12} / \mathrm{L}$ \\
\hline White blood cell count (WBC) & $\begin{array}{l}22.4 \times 10^{-9} / \\
L\end{array}$ & $\begin{array}{l}3.5-9.5 \times 10^{-} \\
9 / \mathrm{L}\end{array}$ \\
\hline Absolute value of neutrophils & $\begin{array}{l}9.46 \times 10^{-9} / \\
L\end{array}$ & $\begin{array}{l}1.8-6.3 \times 10^{-} \\
9 / L\end{array}$ \\
\hline Hemoglobin (HB) & $70 \mathrm{~g} / \mathrm{L}$ & $110-160 \mathrm{~g} / \mathrm{L}$ \\
\hline C-reactive protein (CRP) & $80.86 \mathrm{mg} / \mathrm{L}$ & $0-5 \mathrm{mg} / \mathrm{L}$ \\
\hline Erythrocyte sedimentation rate (ESR) & $4 \mathrm{~mm} / \mathrm{h}$ & $0-20 \mathrm{~mm} / \mathrm{h}$ \\
\hline $\begin{array}{l}\text { Complement hemolytic activity assay } \\
\text { (CH50) }\end{array}$ & $\begin{array}{l}60.0 \mathrm{CH} 50 / \\
\mathrm{ML}\end{array}$ & 80-160.0/ML \\
\hline Interferon- $\gamma($ IFN-- $\gamma)$ & $35.53 \mathrm{pg} / \mathrm{ml}$ & $\begin{array}{l}0.00-2.10 \mathrm{pg} / \\
\mathrm{ml}\end{array}$ \\
\hline Tumor necrosis factor (TNF) & $57.84 \mathrm{pg} / \mathrm{ml}$ & $\begin{array}{l}130-8.55 \mathrm{pg} / \\
\mathrm{ml}\end{array}$ \\
\hline Interleukin 10 (IL-10) & $67.32 \mathrm{pg} / \mathrm{ml}$ & $1.20-4.5 \mathrm{pg} / \mathrm{ml}$ \\
\hline Interleukin 6 (IL-6) & $\begin{array}{l}115.40 \mathrm{pg} / \\
\mathrm{ml}\end{array}$ & $\begin{array}{l}0.00-4.55 \mathrm{pg} / \\
\mathrm{ml}\end{array}$ \\
\hline Interleukin 4 (IL-4) & $0.00 \mathrm{pg} / \mathrm{ml}$ & $\begin{array}{l}1.10-3.65 \mathrm{pg} / \\
\mathrm{ml}\end{array}$ \\
\hline Total protein & $51.8 \mathrm{~g} / \mathrm{L}$ & $62-83 \mathrm{~g} / \mathrm{L}$ \\
\hline Albumin & $29.7 \mathrm{~g} / \mathrm{L}$ & $35-50 \mathrm{~g} / \mathrm{L}$ \\
\hline Globulin & $22.1 \mathrm{~g} / \mathrm{L}$ & $20-40 \mathrm{~g} / \mathrm{L}$ \\
\hline Alanine aminotransferase (ALT) & $123 \mathrm{U} / \mathrm{L}$ & $9-52 \mathrm{U} / \mathrm{L}$ \\
\hline Aspartate aminotransferase (AST) & $113 \mathrm{U} / \mathrm{L}$ & $14-36 \mathrm{U} / \mathrm{L}$ \\
\hline
\end{tabular}

data were compared to reference sequences using the BWA algorithm (UCSC hg19) by instrument default setting [4]. Data was annotated using literature reported methods [5]. After the screening process, bioinformatics software (PolyPhen2, LRT, Mutation Taste, et al.) was used to predict the results. The function, variation, and genetic pattern of each gene was analyzed, and suspicious candidate mutations were obtained. Amplification of suspected candidate mutation sites with PCR primers and Sanger sequencing, at the same time, enabled the corresponding loci of the patient's parents to be detected.

\section{Literature search}

In this study, information about $\mathrm{CD}$ was searched and collected from PubMed, CNKI, Wan Fang Date, and the Human Gene Mutation Database9 (HGMD). Searching terms included "neonate", "Crohn's disease", "inflammatory bowel disease", "interleukin-10 receptor gene (IL10R)", "gene mutation", "oral ulcer", and "perianal disease" as keywords. A list and comparison of 24 cases (including this case report) of IL-10R mutations were

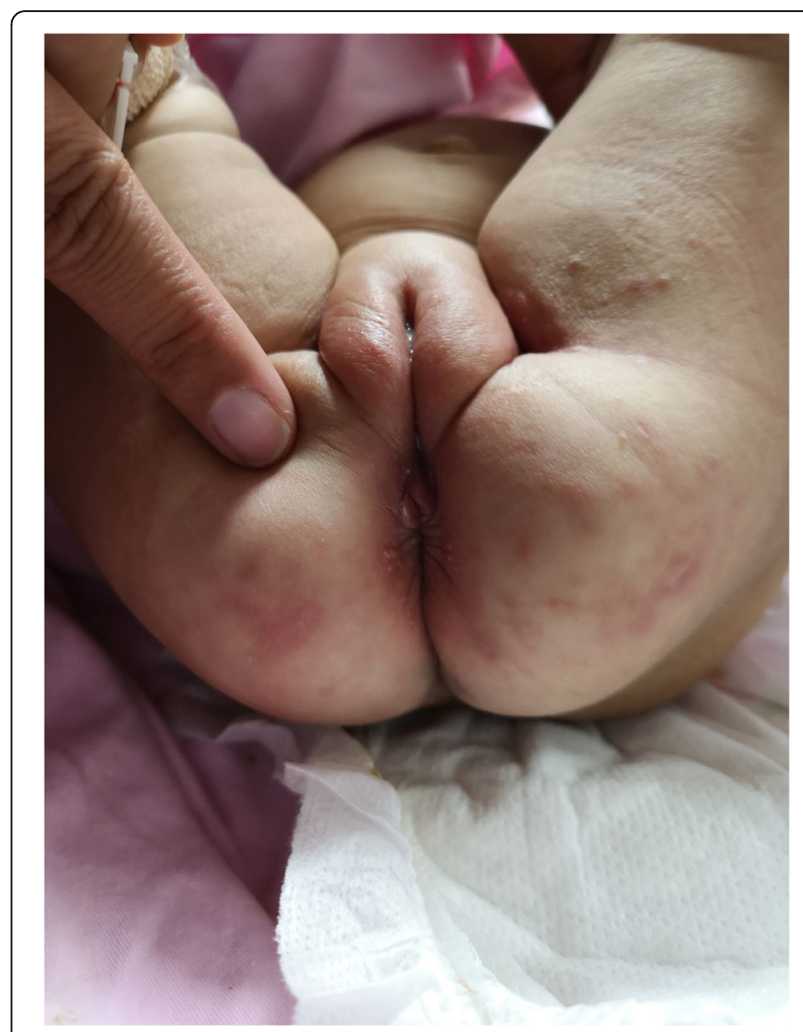

Fig. 2 The protruding masses can be seen around the anus. In addition, a rash can be seen around the anus and inguinal region

identified by genetic analyzing. The information and clinical features of the patients are shown in Table 2.

\section{Results}

\section{Case findings}

NGS identified a compound heterozygous mutation in the interleukin-10 receptor A (IL-10RA) (NM_001558.3) in this patient. One heterozygous mutation was c.301 c > T, P. (Arg 101 Trp) in exon 3 of the IL-10RA gene, which is a missense mutation (Fig. 3a). c.301 c $>$ T, P. (Arg $101 \mathrm{Trp}$ ) has been previously reported as a suspicious pathogenicity mutation $[12,13]$, which results in the conversion of the 101st amino acid Arg to Trp. The second heterozygous mutation was c. $537 \mathrm{G}>\mathrm{A}$, P. (Thr $179=$ ) in exon 4 of the IL-10RA gene, which is a synonymous mutation (Fig. 4a). c. $537 \mathrm{G}>\mathrm{A}, \mathrm{P}$. (Thr $179=)$ has previously been reported as a pathogenicity mutation [13], and can affect mRNA splicing.

In order to investigate the genetic pattern, the patient's parents also underwent NGS. The results showed that her father carries one heterozygous mutation: c.301 c > T, P. (Arg 101 Trp) in exon 3 of the IL-10RA gene (Fig. $3 \mathrm{~b})$. Furthermore, her mother also carries one heterozygous mutation: c.537G > A, P. (Thr $179=$ ) in exon 4 of the IL-10RA gene (Fig. 4c). The results showed that 
Table 2 Literature search results

\begin{tabular}{|c|c|c|c|c|c|}
\hline Patients & $\begin{array}{l}\text { Age/gender } \\
\text { of onset }\end{array}$ & $\begin{array}{l}\text { Mutation gene/type } \\
\text { site/amino acid } \\
\text { change }\end{array}$ & Clinical outcome & Parent gene & $\begin{array}{l}\text { Author/ } \\
\text { reference }\end{array}$ \\
\hline 1 & $30 \mathrm{~d} /$ female & $\begin{array}{l}\text { IL-10RA / Homozygous } \\
\text { C.537G > A / P.179T > T }\end{array}$ & $\begin{array}{l}\text { Clinical: Fever, diarrhea, bloody mucous stool, oral ulcers, } \\
\text { anemia. } \\
\text { ED: Scattered ulcers in colon and rectum. } \\
\text { HD: Ulcers with chronic inflammatory cells and neutrophil } \\
\text { infiltration. } \\
\text { CRP: } 71.4 \mathrm{mg} / \mathrm{L} ; \text { ESR: } 58 \mathrm{~mm} / 1 \mathrm{~h}\end{array}$ & $\begin{array}{l}F: N R \\
M: N R\end{array}$ & $\begin{array}{l}\text { Wang FP, } \\
\text { et al. [2018] } \\
\text { [6] }\end{array}$ \\
\hline 2 & $10 \mathrm{~d} /$ female & $\begin{array}{l}\text { IL-10RA / Homozygous } \\
\text { c.301C }>\text { T / P.101R }>\text { W }\end{array}$ & $\begin{array}{l}\text { Clinical: Fever, diarrhea, mucous stool, rectovaginal fistula, } \\
\text { anemia, innutrition, hypoproteinemia. } \\
\text { ED: Multiple ulcers and polyp in ileocecum, colon, and } \\
\text { rectum; segment presentation. } \\
\text { HD: Acute and chronic inflammatory cell infiltration and } \\
\text { granulation tissue. } \\
\text { CRP: } 22.0 \mathrm{mg} / \mathrm{L} \text {; ESR: } 11 \mathrm{~mm} / 1 \mathrm{~h}\end{array}$ & $\begin{array}{l}\text { F: NR } \\
M: N R\end{array}$ & $\begin{array}{l}\text { Wang FP, } \\
\text { et al. [2018] } \\
\text { [6] }\end{array}$ \\
\hline 3 & $1 \mathrm{~d} /$ female & $\begin{array}{l}\text { IL-10RA / Homozygous } \\
\text { C.301C }>\text { T / P.101R }>\text { W }\end{array}$ & $\begin{array}{l}\text { Clinical: Fever, diarrhea, perianal fistulas, anemia, innutrition, } \\
\text { and hypoproteinemia. } \\
\text { ED: Scattered ulcers in colon and rectum. } \\
\text { HD: Acute and chronic inflammatory cell infiltration and } \\
\text { mucosal erosive ulcer. } \\
\text { CRP: } 5.0 \mathrm{mg} / \mathrm{L} \text {; ESR: } 34 \mathrm{~mm} / 1 \mathrm{~h}\end{array}$ & $\begin{array}{l}\text { F: NR } \\
\text { M: NR }\end{array}$ & $\begin{array}{l}\text { Wang FP, } \\
\text { et al. [2018] } \\
\text { [6] }\end{array}$ \\
\hline 4 & Days /male & $\begin{array}{l}\text { IL-10RB / Compound } \\
\text { heterozygous mutation } \\
\text { c. } 301 \mathrm{C}>\mathrm{T} / \mathrm{P} \text {. } \\
101 \mathrm{R}>\mathrm{W} \\
\text { c.537 G }>\text { A / P.179 T > } \\
\mathrm{T}\end{array}$ & $\begin{array}{l}\text { Clinical: Fistulas fever, diarrhea, perianal ulcer, anemia, } \\
\text { innutrition, hypoproteinemia. } \\
\text { ED: Irregular ulcers and false polyps in ileocecum, colon, } \\
\text { and rectum. } \\
\text { HD: Acute and chronic inflammatory cell infiltration with } \\
\text { crypt inflammation and crypt abscess. } \\
\text { CRP: } 68.7 \mathrm{mg} / \mathrm{L} \text {; ESR: } 17 \mathrm{~mm} / 1 \mathrm{~h}\end{array}$ & $\begin{array}{l}\text { F: NR } \\
\text { M: NR }\end{array}$ & $\begin{array}{l}\text { Wang FP, } \\
\text { et al. [2018] } \\
\text { [6] }\end{array}$ \\
\hline 5 & $1 \mathrm{~d} /$ male & $\begin{array}{l}\text { IL-10RA / Compound } \\
\text { heterozygous mutation } \\
\text { C. } 301 \mathrm{C}>\mathrm{T} / \mathrm{P} \text {. } \\
101 \mathrm{R}>\mathrm{W} \\
\text { C. } 350 \mathrm{G}>\mathrm{A} / \mathrm{P} .117 \mathrm{R}> \\
\mathrm{H}\end{array}$ & $\begin{array}{l}\text { Clinical: Fever, diarrhea, oral ulcer, } \\
\text { anemia, perianal neoplasm, innutrition, hypoproteinemia. } \\
\text { ED: Scattered ulcers in colon and rectum. } \\
\text { HD: Acute and chronic inflammatory cell infiltration with } \\
\text { ulcer and crypt abscess. } \\
\text { CRP: } 23.1 \mathrm{mg} / \mathrm{L} \text {; ESR: ESR:29 mm/1 h }\end{array}$ & $\begin{array}{l}\text { F: NR } \\
\text { M: NR }\end{array}$ & $\begin{array}{l}\text { Wang FP, } \\
\text { et al. [2018] } \\
\text { [6] }\end{array}$ \\
\hline 6 & $16 \mathrm{~d} /$ female & $\begin{array}{l}\text { IL-10RA / Compound } \\
\text { heterozygous mutation } \\
\text { C. } 301 \mathrm{C}>\mathrm{T} / \mathrm{P} \text {. } \\
101 \mathrm{R}>\mathrm{W} \\
\text { C. } 299 \mathrm{~T}>\mathrm{G} / \mathrm{P} .100 \mathrm{~V}> \\
\mathrm{G}\end{array}$ & $\begin{array}{l}\text { Clinical: Fever, diarrhea, bloody purulent stool, perianal } \\
\text { fistulas, anemia, innutrition, hypoproteinemia. } \\
\text { ED: Scattered ulcers in colon and rectum. } \\
\text { HD: Acute and chronic inflammatory cell infiltration with } \\
\text { crypt inflammation and crypt abscess. } \\
\text { CRP: } 27.9 \mathrm{mg} / \mathrm{L} \text {; ESR: } 65 \mathrm{~mm} / 1 \mathrm{~h}\end{array}$ & $\begin{array}{l}\text { F: NR } \\
\text { M: NR }\end{array}$ & $\begin{array}{l}\text { Wang FP, } \\
\text { et al. [2018] } \\
\text { [6] }\end{array}$ \\
\hline 7 & NR /male & $\begin{array}{l}\text { IL-10RA / Compound } \\
\text { heterozygous mutation } \\
\text { c. } 537 \mathrm{G}>\text { A / P. C } 223 \text { S } \\
\text { c. } 668 \mathrm{G}>\text { C }\end{array}$ & $\begin{array}{l}\text { Clinical: Fever, diarrhea, bloody purulent stool, erythra in } \\
\text { eye lid and back. } \\
\text { ED: NR } \\
\text { HD: NR. } \\
\text { CRP: NR; ESR: NR }\end{array}$ & $\begin{array}{l}\text { F: NR } \\
M: N R\end{array}$ & $\begin{array}{l}\text { Lu D, et al. } \\
\text { [2015] [7] }\end{array}$ \\
\hline 8 & NR /male & $\begin{array}{l}\text { IL-10RA / Compound } \\
\text { heterozygous mutation } \\
\text { c. } 537 \mathrm{G}>\mathrm{A} \text { / P. C } 223 \mathrm{~S} \\
\text { c. } 668 \mathrm{G}>\mathrm{C}\end{array}$ & $\begin{array}{l}\text { Clinical: Mucosanguineous feces, perianal abscesses, } \\
\text { neoplasm. } \\
\text { ED: NR } \\
\text { HD: NR } \\
\text { CRP: NR; ESR: NR }\end{array}$ & $\begin{array}{l}\text { F: NR } \\
\text { M: NR }\end{array}$ & $\begin{array}{l}\text { Lu D, et al. } \\
\text { [2015] [7] }\end{array}$ \\
\hline 9 & $4 \mathrm{~d} /$ female & $\begin{array}{l}\text { IL-10RA / Compound } \\
\text { heterozygous mutation } \\
\text { C. } 421 \mathrm{G}>\mathrm{A} / \mathrm{P} \text {. } \\
141 \mathrm{G}>\mathrm{W} \\
\text { C. } 301 \mathrm{C}>\mathrm{T} / \mathrm{P} .101 \mathrm{R}> \\
\text { W }\end{array}$ & $\begin{array}{l}\text { Clinical: Fever, diarrhea, bloody stool, oral ulcers, anal } \\
\text { fissure, hypoproteinemia, hepatosis } \\
\text { ED: Extensive ulcers in colon, ileocecal erosion. } \\
\text { HD: chronic inflammatory infiltration with crypt } \\
\text { inflammation. } \\
\text { CRP: +; ESR: - }\end{array}$ & $\begin{array}{l}\text { F: NR } \\
M: N R\end{array}$ & $\begin{array}{l}\text { Jiang } Y \text {, et al. } \\
\text { [2017] [8] }\end{array}$ \\
\hline 10 & $14 \mathrm{~d} /$ male & $\begin{array}{l}\text { IL-10RA / Homozygous } \\
\text { C.537 G > A / P.179T> } \\
\mathrm{T}\end{array}$ & $\begin{array}{l}\text { Clinical: Fever, diarrhea, bloody stool, oral ulcer. } \\
\text { ED: Ulcers in colon. } \\
\text { HD: Diffuse lymphocytic infiltration in ulcer. } \\
\text { CRP: -; ESR: + }\end{array}$ & $\begin{array}{l}F: N R \\
M: N R\end{array}$ & $\begin{array}{l}\text { Jiang } Y \text {, et al. } \\
\text { [2017] [8] }\end{array}$ \\
\hline 11 & $10 \mathrm{~d} /$ female & $\begin{array}{l}\text { IL-10RA / Compound } \\
\text { heterozygous mutation }\end{array}$ & $\begin{array}{l}\text { Clinical: Fever, diarrhea, mucous bloody stool, anal fissure, } \\
\text { Perianal lesion, erythra, hepatosis. }\end{array}$ & $\begin{array}{l}F: N R \\
M: N R\end{array}$ & $\begin{array}{l}\text { Jiang Y, et al. } \\
{[2017][8]}\end{array}$ \\
\hline
\end{tabular}


Table 2 Literature search results (Continued)

\begin{tabular}{|c|c|c|c|c|c|}
\hline Patients & $\begin{array}{l}\text { Age/gender } \\
\text { of onset }\end{array}$ & $\begin{array}{l}\text { Mutation gene/type } \\
\text { site/amino acid } \\
\text { change }\end{array}$ & Clinical outcome & Parent gene & $\begin{array}{l}\text { Author/ } \\
\text { reference }\end{array}$ \\
\hline & & $\begin{array}{l}X \\
\text { C.301C > T / P.101R> } \\
W\end{array}$ & $\begin{array}{l}\text { HD: chronic inflammatory with crypt inflammation and } \\
\text { perianal neoplasm } \\
\text { CRP: +; ESR: - }\end{array}$ & & \\
\hline 12 & $14 \mathrm{~d} /$ female & $\begin{array}{l}\text { IL-10RA / Compound } \\
\text { heterozygous mutation } \\
\text { C. } 537 \mathrm{G}>\text { A / P. } 179 \\
\text { T }>\text { T } \\
\text { c.301C > T / P.101 R > } \\
\text { W }\end{array}$ & $\begin{array}{l}\text { Clinical: Fever, diarrhea, bloody stool, oral ulcers, anal } \\
\text { fissure, perianal fistulas. } \\
\text { ED: Extensive ulcers in colon, ileocecal terminal erosion. } \\
\text { HD: Acute and chronic inflammatory cell infiltration with } \\
\text { crypt inflammation and crypt abscess. } \\
\text { CRP: +; ESR: - }\end{array}$ & $\begin{array}{l}F: N R \\
M: N R\end{array}$ & $\begin{array}{l}\text { Jiang } Y \text {, et al. } \\
{[2017][8]}\end{array}$ \\
\hline 13 & $9 \mathrm{~d} /$ male & $\begin{array}{l}\text { IL-10RA / Compound } \\
\text { heterozygous mutation } \\
\text { C. } 537 \mathrm{G}>\text { A / P. } 179 \\
\text { T }>\text { T } \\
\text { c.301C > T / P.101 R > } \\
\text { W }\end{array}$ & $\begin{array}{l}\text { Clinical: Fever, diarrhea, bloody stool, oral ulcer, perianal } \\
\text { neoplasm. } \\
\text { ED: Ulcers in transverse colon. } \\
\text { HD: Chronic inflammation of small and large intestine } \\
\text { mucosa with crypt inflammation. } \\
\text { CRP: +; ESR: - }\end{array}$ & $\begin{array}{l}F: N R \\
M: N R\end{array}$ & $\begin{array}{l}\text { Jiang } Y \text {, et al. } \\
{[2017][8]}\end{array}$ \\
\hline 14 & NR /female & $\begin{array}{l}\text { IL-10RA / Compound } \\
\text { heterozygous mutation } \\
\text { C. 301C > T / P.R101 W } \\
\text { C.350G > A / P.R } 117 \text { H }\end{array}$ & $\begin{array}{l}\text { Clinical: Diarrhea, perianal fistulas. } \\
\text { ED: NR. } \\
\text { HD: NR. } \\
\text { CRP: NR; ESR: NR }\end{array}$ & $\begin{array}{l}F: N R \\
M: N R\end{array}$ & $\begin{array}{l}\text { Shim JO, } \\
\text { et al. [2013] } \\
\text { [9] }\end{array}$ \\
\hline 15 & NR /male & $\begin{array}{l}\text { IL-10RA / Compound } \\
\text { heterozygous mutation } \\
\text { C. } 272 \mathrm{~A}>\mathrm{G} \mathrm{G} \mathrm{P.Y91C} \\
\text { C.784C > A / R } 262 \text { C }\end{array}$ & $\begin{array}{l}\text { Clinical: Diarrhea, perianal fistulas, intestinal fistula. } \\
\text { ED: NR. } \\
\text { HD: NR. } \\
\text { CRP: NR; ESR: NR }\end{array}$ & $\begin{array}{l}F: N R \\
M: N R\end{array}$ & $\begin{array}{l}\text { Shim JO, } \\
\text { et al. [2013] } \\
{[9]}\end{array}$ \\
\hline 16 & NR /female & $\begin{array}{l}\text { IL-10RA/Compound } \\
\text { heterozygous mutation } \\
\text { C. } 272 \mathrm{~A}>\mathrm{G} / \text { P.Y91C } \\
\text { C.301 C> T /.R } 101 \mathrm{~W}\end{array}$ & $\begin{array}{l}\text { Clinical: Diarrhea, perianal fistulas, intestinal fistula. } \\
\text { ED: NR. } \\
\text { HD: NR. } \\
\text { CRP: NR; ESR: NR }\end{array}$ & $\begin{array}{l}F: N R \\
M: N R\end{array}$ & $\begin{array}{l}\text { Shim JO, } \\
\text { et al. [2013] } \\
\text { [9] }\end{array}$ \\
\hline 17 & $\begin{array}{l}\text { Neonate } \\
\text { /male }\end{array}$ & $\begin{array}{l}\text { IL-10RA / Homozygous } \\
\text { C. } 537 G>\text { A / P. } 179 \\
T>T \\
\text { C. } 301 C>T / P .101 \text { R }> \\
W\end{array}$ & $\begin{array}{l}\text { Clinical: Fever, diarrhea, bloody stool, anal fissure, perianal } \\
\text { abscesses, skin infections. } \\
\text { ED: Irregular ulcers and polyps in colon. } \\
\text { HD: Inflammatory cell infiltration with ulcer. } \\
\text { CRP: NR; ESR: NR }\end{array}$ & $\begin{array}{l}\text { F: Homozygous } \\
\text { C.737G > A } \\
\text { M: NR }\end{array}$ & $\begin{array}{l}\text { Fang } Y H, \\
\text { et al. [2018] } \\
\text { [10] }\end{array}$ \\
\hline 18 & $\begin{array}{l}4 \text { months } \\
\text { /female }\end{array}$ & $\begin{array}{l}\text { IL-10RA / Homozygous } \\
\text { C. 301C > T / P.R101W } \\
\text { c.537G > A / PT179 T }\end{array}$ & $\begin{array}{l}\text { Clinical: Fever, diarrhea, mucous bloody stool, perianal } \\
\text { fistulas. } \\
\text { ED: Many ulcers and a cobblestone-like vegetation in colon. } \\
\text { HD: Inflammatory cell infiltration in ulcer. } \\
\text { CRP: NR; ESR: NR }\end{array}$ & $\begin{array}{l}\text { F: Homozygous } \\
\text { C.737G > A } \\
\text { M: Homozygous } \\
\text { P > T179T }\end{array}$ & $\begin{array}{l}\text { Fang YH, } \\
\text { et al. [2018] } \\
{[10]}\end{array}$ \\
\hline 19 & $\begin{array}{l}\text { More than } 1 \\
\text { month } \\
\text { /female }\end{array}$ & $\begin{array}{l}\text { IL-10RA / Homozygous } \\
\text { C. } 301 \mathrm{C}>\mathrm{T} / \text { P.R101W } \\
\text { c. } 470 \mathrm{~A}>\mathrm{G} / \mathrm{P.Y157C}\end{array}$ & $\begin{array}{l}\text { Clinical: Diarrhea, mucous bloody stool, perianal fistulas, oral } \\
\text { ulcer, innutrition, hypoevolutism. } \\
\text { ED: Colon ulcers. } \\
\text { HD: ND } \\
\text { CRP: NR; ESR: NR }\end{array}$ & $\begin{array}{l}\text { F: Homozygous } \\
\text { C.301C > T } \\
\text { M: Homozygous } \\
\text { C. } 470 \mathrm{~A}>\mathrm{G}\end{array}$ & $\begin{array}{l}\text { Fang YH, } \\
\text { et al. [2018] } \\
\text { [10] }\end{array}$ \\
\hline 20 & $\begin{array}{l}\text { More than } 1 \\
\text { month /male }\end{array}$ & $\begin{array}{l}\text { IL-10RA / Homozygous } \\
\text { C. 301C > T / P.R101W }\end{array}$ & $\begin{array}{l}\text { Clinical: Bloody stool, erythra, repeated perianal abscess, } \\
\text { innutrition. } \\
\text { ED: Irregular ulcers and polyps in colon sigmoideum and } \\
\text { rectum. } \\
\text { HD: NR } \\
\text { CRP: NR; ESR: NR }\end{array}$ & $\begin{array}{l}\text { F: Homozygous } \\
\text { C.301C > T } \\
\text { M: NR }\end{array}$ & $\begin{array}{l}\text { Fang YH, } \\
\text { et al. [2018] } \\
\text { [10] }\end{array}$ \\
\hline 21 & $\begin{array}{l}\text { Neonate } \\
\text { /male }\end{array}$ & $\begin{array}{l}\text { IL-10RA / Compound } \\
\text { heterozygous mutation } \\
\text { C. 301C > T / P.R101W } \\
\text { C.350G > A / P.R117H }\end{array}$ & $\begin{array}{l}\text { Clinical: Bloody stool, perianal fistulas, necrotizing } \\
\text { enterocolitis, innutrition. } \\
\text { ED: Many ulcers in rectum and polyps in colon. } \\
\text { HD: ND. } \\
\text { CRP: NR; ESR: NR }\end{array}$ & $\begin{array}{l}\text { F: Compound } \\
\text { heterozygous } \\
\text { mutation } \\
\text { C. } 301 C>\text { T /P.R101W } \\
\text { C.350G > A/P.R117H } \\
\text { M: NR }\end{array}$ & $\begin{array}{l}\text { Fang } \mathrm{YH}_{1} \\
\text { et al. [2018] } \\
\text { [10] }\end{array}$ \\
\hline 22 & $\begin{array}{l}5 \text { years } 9 \\
\text { months } \\
\text { /female }\end{array}$ & $\begin{array}{l}\text { IL-10RB / heterozygous } \\
\text { C. } 421 \mathrm{G}>\mathrm{A} / \mathrm{P} .141 \mathrm{~K}\end{array}$ & $\begin{array}{l}\text { Clinical: NR. } \\
\text { ED: NR. } \\
\text { HD: NR } \\
\text { CRP: NR; ESR: NR }\end{array}$ & $\begin{array}{l}\text { F: Heterozygous } \\
\text { C. } 421 \mathrm{G}>\mathrm{A} \\
\text { M: NR }\end{array}$ & $\begin{array}{l}\text { Fang YH, } \\
\text { et al. [2018] } \\
\text { [10] }\end{array}$ \\
\hline 23 & NR /male & $\begin{array}{l}\text { IL-10RA / NR } \\
\text { c.537G > A / P.T179 T }\end{array}$ & $\begin{array}{l}\text { Clinical: Diarrhea, bloody stool, perinanal fistulas, oral ulcers, } \\
\text { epifolliculitis. }\end{array}$ & $\begin{array}{l}F: N R \\
M: N R\end{array}$ & $\begin{array}{l}\text { Yanagi T, } \\
\text { et al. [2016] }\end{array}$ \\
\hline
\end{tabular}


Table 2 Literature search results (Continued)

\begin{tabular}{|c|c|c|c|c|c|}
\hline Patients & $\begin{array}{l}\text { Age/gender } \\
\text { of onset }\end{array}$ & $\begin{array}{l}\text { Mutation gene/type } \\
\text { site/amino acid } \\
\text { change }\end{array}$ & Clinical outcome & Parent gene & $\begin{array}{l}\text { Author/ } \\
\text { reference }\end{array}$ \\
\hline & & & $\begin{array}{l}\text { ED: Longitudinal ulcers in colon. } \\
\text { HD: NR. } \\
\text { CRP: NR; ESR: NR }\end{array}$ & & [11] \\
\hline 24 & $10 \mathrm{~d} /$ female & $\begin{array}{l}\text { IL-10RA / Compound } \\
\text { heterozygous mutation } \\
\text { c. } 301 \text { C > T / } \\
\text { Arg101Frp } \\
\text { c.537G > A / P.Tnr179 } \\
=\end{array}$ & $\begin{array}{l}\text { Clinical: Oral ulcers, fever, rash, perianal masses, perianal } \\
\text { pyoderma, anemia, innutrition, hypoproteinemia, hepatosis. } \\
\text { ED: ND. } \\
\text { HD: ND } \\
\text { CRP: } 80.86 \mathrm{mg} / \mathrm{L} \text {; ESR: } 4 \mathrm{~mm} / 1 \mathrm{~h}\end{array}$ & $\begin{array}{l}\text { F: Heterozygous } \\
\text { C.301C }>\text { T/ } \\
\text { Arg101Frp } \\
\text { M: Heterozygous } \\
\text { C.537G > A/ P.Tnr179 } \\
=\end{array}$ & [This report] \\
\hline
\end{tabular}

IBD inflammatory bowel disease; $C D$ Crohn's disease; NR not recorded in detail; ND not detected; $E D$ endoscope detection; HD histopathological detection; CRP Creactive protein; ESR erythrocyte sedimentation rate; $F$ father; $M$ mother.; $N R$ normal range

genetic mutations in the following genes were not present in the patient: XIAP, 33 NCF2 34, MEFV35, LRBA, XIAP, TNFR13B, and CYBB.

\section{Literature search findings}

In addition, we collected information on 24 cases (including this case) from previous studies reporting on neonates with $\mathrm{CD}$ or early IBD caused by mutations in L-10 or L-10R. Their clinical manifestations and gene phenotypes are summarized in Table 2 . The results show that the incidence rate of neonatal CD or IBD is equal regarding gender (except for one case with incomplete clinical records), fever occurred in $65.52 \%(15 / 23)$, diarrhea in $82.26 \%(19 / 23)$, mucous stools, bloody purulent stools, or bloody stools in $69.57 \%(16 / 23)$, oral ulcers in $39.13 \%$ (9/23), perianal diseases in $86.96 \%$ (20/23) (including perianal mass/neoplasms, perianal abscesses, perianal ulcer, perianal pyoderma, anal fistulas, anal fissure, and rectovaginal fistula), eczema/ rash in $21.74 \%$ (5/23), anemia in $39.14 \%$ (9/23), hypoproteinemia in $26.09 \%(6 / 23)$, innutrition in $39.14 \%$ (9/23), liver dysfunction in $13.04 \%(3 / 23)$, intestinal fistula in $8.70 \%$ (2/ $23)$, intestinal necrosis in $8.70 \%(2 / 23)$, and folliculitis in $4.35 \%(1 / 23)$ of cases. In 24 cases of IL-10R mutations, IL-10RA mutations accounted for $91.67 \%(22 / 24)$ and IL-10RB mutations accounted for $8.33 \%(2 / 24)$. Regarding the gene mutation types (except for one case where it was not recorded), a compound heterozygous mutation accounted for $69.56 \%(16 / 23)$, a homozygous mutation accounted for $26.09 \%(6 / 23)$, and a heterozygous mutation accounted for $4.35 \%(1 / 23)$. The gene mutation site was c. $301 \mathrm{C}>\mathrm{T}$ in $66.67 \%$ $(16 / 24)$, c.537G $>A$ in $41.67 \%(10 / 24)$, c.350G $>A$ in $12.50 \%(3 / 24)$, c.272 A > G in $10.42 \%(2 / 24)$, c.688G > $\mathrm{C}$ in $10.42 \%(2 / 24), \quad c .421 \mathrm{G}>\mathrm{A}$ in $10.42 \%(2 / 24)$, c. $493 \mathrm{C}>\mathrm{T}$ in $4.17 \%(1 / 24)$, c.737G $>\mathrm{A}$ in $4.17 \%(1 /$ 24), c. $299 \mathrm{~T}>\mathrm{G}$ in $4.17 \%(1 / 24)$, c. $784 \mathrm{C}>\mathrm{T}$ in $4.17 \%$ $(1 / 24)$, and c. $470 \mathrm{~A}>\mathrm{G}$ in $4.17 \%(1 / 24)$.

\section{Discussion}

We report the case of a 10-day-old girl with neonatal $\mathrm{CD}$ diagnosed after genetic sequencing, which revealed a compound heterozygous mutation in the interleukin-10 receptor A (IL-10RA) (NM_001558.3) gene. One heterozygous mutation was c.301 c>T, P. (Arg 101 Trp) in exon 3 of IL-10RA (a missense mutation), and the other was c. $537 \mathrm{G}>\mathrm{A}, \mathrm{P}$. (Thr $179=$ ) in exon 4 of IL 10RA (a synonymous mutation).

IBD includes three subtypes: ulcerative colitis, CD, and indeterminate colitis (IC). CD was first described by Crohn, Ginzterg, and Oppenheime in 1932. In IBD cases, $\mathrm{CD}$ is more common in children, but ulcerative colitis is more common in adults [14]. The prevalence of $\mathrm{CD}$ is $30-35 \%$ in children with very early inflammatory bowel disease (VIBD) [15].

Thus far, the etiology and mechanism of CD are not well understood. It is considered that $\mathrm{CD}$ is related to intestinal flora imbalance, immune system interactions, and genetic mutations [16]. Epidemiological investigations have shown that early intrauterine infection is associated with $\mathrm{CD}$; in particular, the measles virus infection is an important risk factor [17]. However, other studies have failed to show an association [18]. In recent years, some scholars have found that the occurrence of $\mathrm{CD}$ is related to the birth season, as the risk of CD was reported to be higher for those born in the first half of the year [19]. In contrast, another study reported that the highest risk of CD is in those born in the second half of the year, not the first [20].

The NOD2/CARD15 gene was discovered as the first susceptible gene in $\mathrm{CD}$. As a result, the relationship between gene variation and $C D$ has attracted much attention. The proband concordance rate among monozygotic twins is $6.3 \%$ for ulcerative colitis and $58.3 \%$ for CD [21]. These results indicated that the incidence rate of monozygotic pairs in $\mathrm{CD}$ is significantly higher than that of dizygotic twins; therefore, it is suggested that 


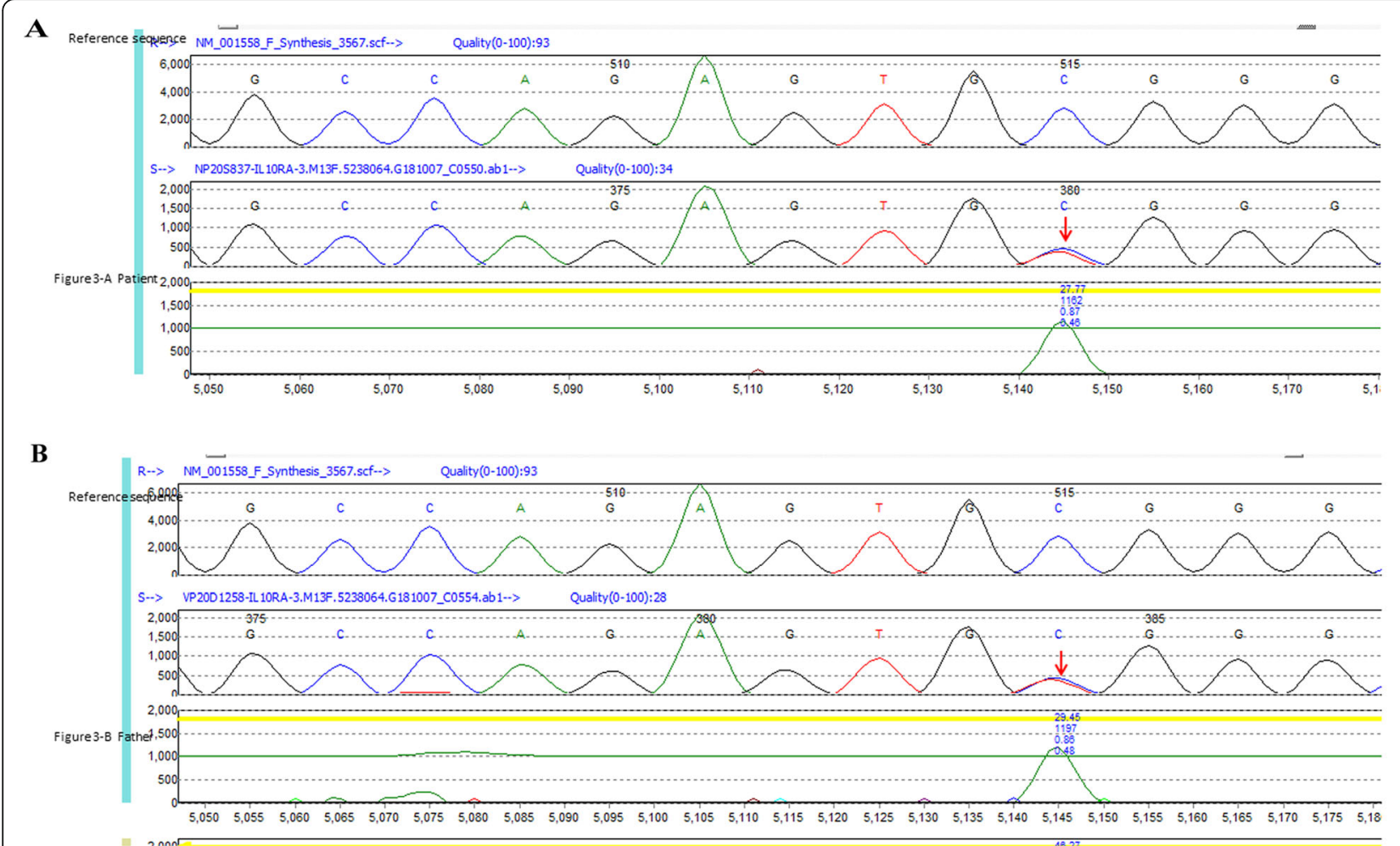

C Reference sequence

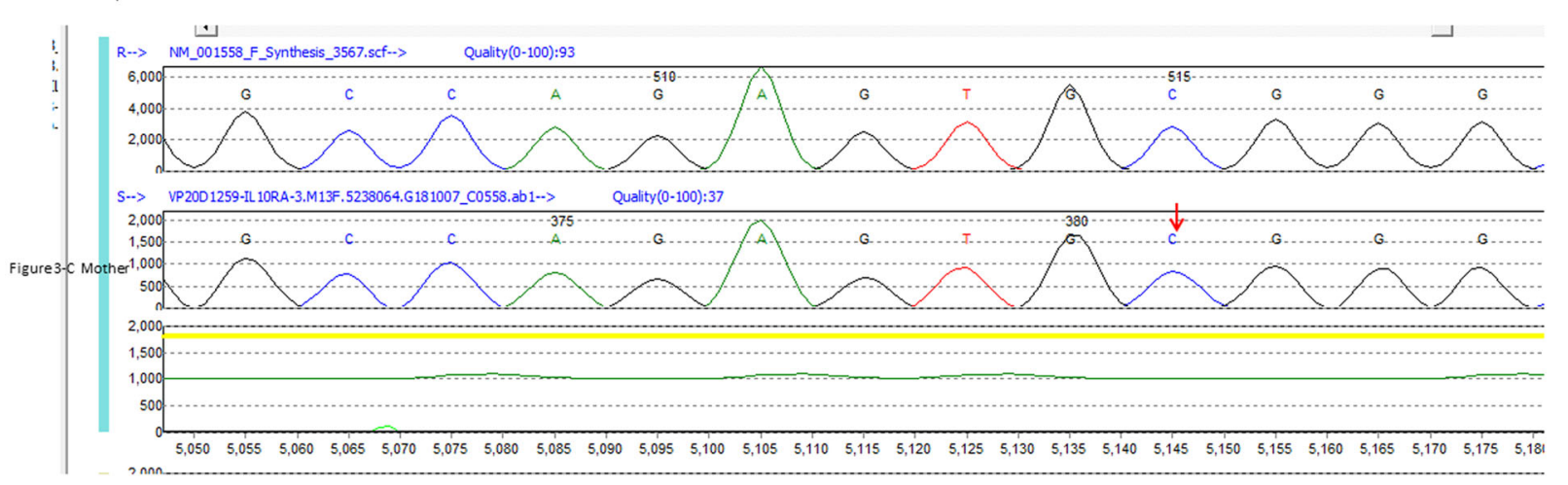

Fig. 3 The patient carries one heterozygous mutation, c.301 c> T, P. (Arg 101 Trp), in exon 3 of the IL-10RA gene, which is a missense mutation (a, arrow). Her father also carries one heterozygous mutation, c.301 c> T, P. (Arg 101 Trp), in exon 3 of the IL-10RA gene (b, arrow). Her mother has no abnormalities in this coding region of the IL-10RA gene (3-c)

heredity factors play an important role in the pathogenesis of CD. Many epidemiological studies have shown that the occurrence of $\mathrm{CD}$ is related to many susceptible genes. With the rapid development and clinical application of genetics technology, the accuracy of neonatal CD diagnosis has greatly improved. To date, the known susceptibility genes for neonatal CD or VIBD include XIAP, 33 NCF2 34, MEFV35, LRBA, IL10 5, IL-10RA, XIAP, TNFRF13B, CYBB [22], and ABCB1 gene mutations [23]. However, the mechanisms by which these gene mutations lead to neonatal CD or VIBD are not yet clear. It is believed that these susceptible genes are involved in maintaining epithelial barrier function, affecting the phagocytosis of monocytes and granulocytes, and can also affect the balance between the proinflammatory and anti-inflammatory response [24]. Glocker EO et al. were the first to discover that mutations in genes encoding the $\alpha$-subunit (IL-10R1, encoding gene IL-10RA) and the $\beta$-subunit (IL-10R2, encoding gene IL-10RB) of the interleukin-10 (IL-10) receptor could induce VIBD development [25]. These results have since aroused great interest for many scholars. Further study has confirmed IL-10 and IL-10 receptor gene mutations to be related to the phenotype of severe perianal 

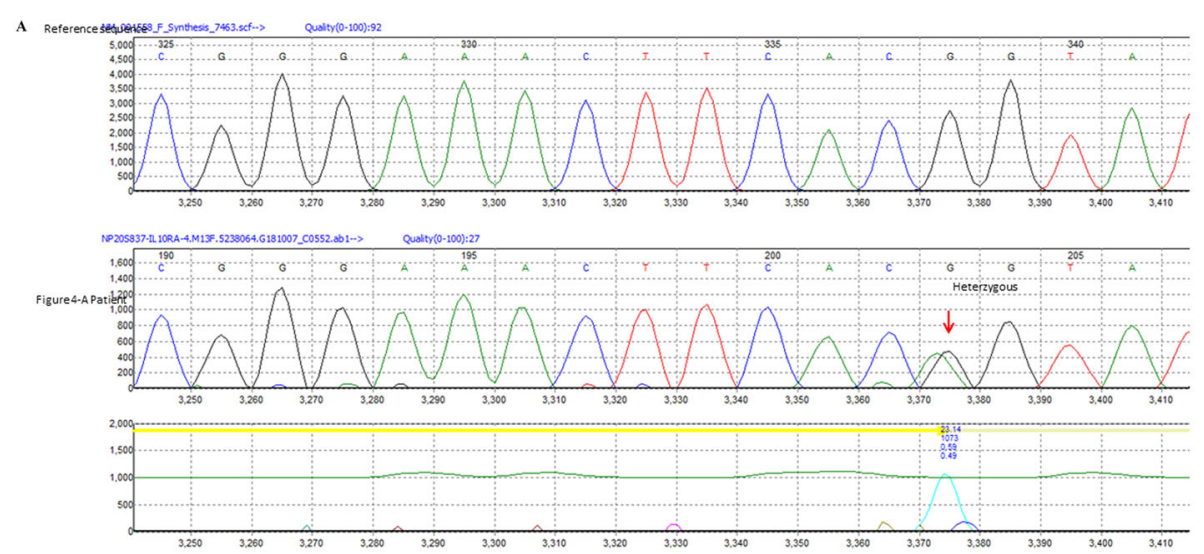

B
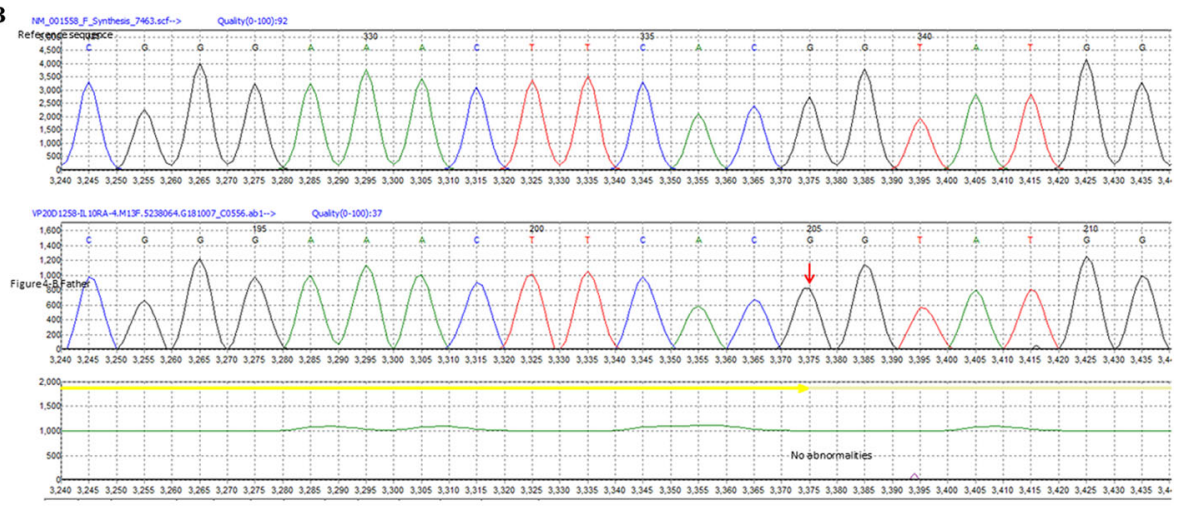

C
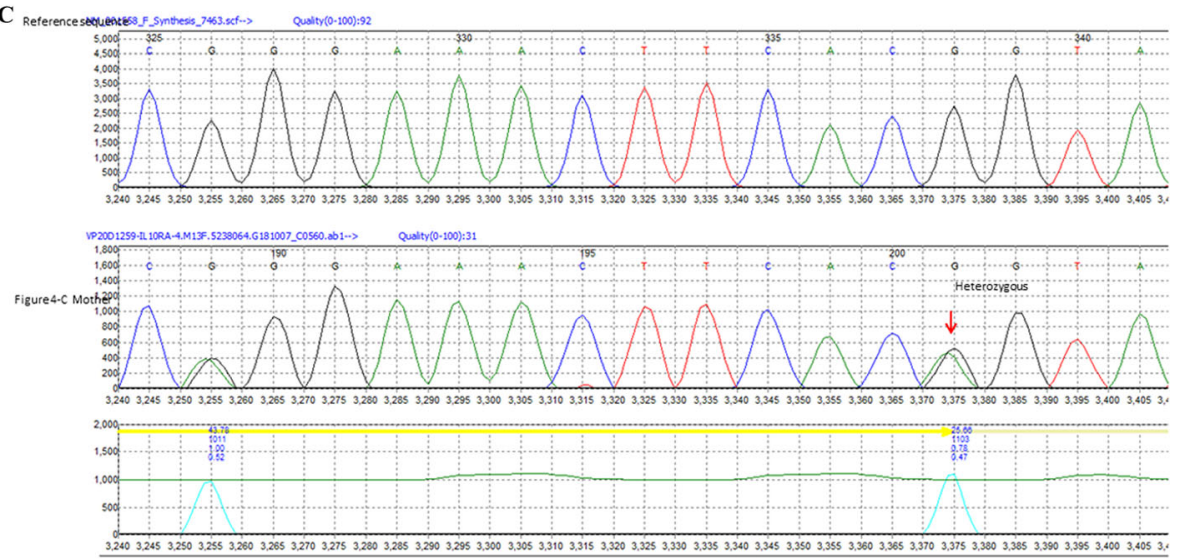

Fig. 4 The patient carries one heterozygous mutation, c. 537G > A, P. (Thr $179=$ ), in exon 4 of the IL-10RA gene, which is a synonymous mutation (a, arrow). Her father has no abnormalities in this coding region of the IL-10RA gene (b). However, her mother carries one heterozygous mutation, C.537G > A, P. (Thr $179=$ ), in exon 4 of the IL-10RA gene (c, arrow)

diseases and VIBD, particularly in infants [26, 27], and to be the main cause of neonatal $\mathrm{CD}$ and enterocolitis $[6-10,24,28,29]$. In the present study, the patient was eventually diagnosed with neonatal $\mathrm{CD}$ caused by a compound heterozygous mutation of the IL-10RA gene. However, laboratory tests showed that the patient had elevated levels of interferon- $\gamma$, C-reactive protein, IL-6, and IL-10 (Table 1). Based on this data, we speculate that the compound heterozygous mutation of the IL10RA gene can lead to a dysfunction in the immune system, which develops a series of clinical manifestations of $\mathrm{CD}$.

According to the type of gene structure change, gene mutations can be divided into four categories: base substitution, frame shift mutation, deletion mutation, and insertion mutation. According to the change in genetic information, gene mutations can be classified into three types: synonymous mutation, missense mutation, and nonsense mutation. Kelsen JR et al. reported on heterozygous missense variants of IL10RA and unidentified 
variants of MSH5 and CD19 in early IBD in newborns [30]. Huang $\mathrm{Z}$ et al. found that C301C $>\mathrm{T}$ (p.R101RW) and c.537G > A (PT179 T) were the most common mutations of the IL-10RA gene, accounting for $88.1 \%$ of all neonatal $\mathrm{CD}$ patients [31]. Yanagi $\mathrm{T}$ et al. reported a new mutation (c. $537 \mathrm{G}>$ AgnpT179 T) in exon 4 of the IL-10RA gene, resulting in unique splicing aberration, with a lack of signaling of the IL-10 receptor; the patient also developed immune thrombocytopenic purpura and transient features mimicking juvenile myelomonocytic leukemia [11]. Our patient carried a compound heterozygous mutation in the IL-10 RA, one heterozygous mutation occurred (c.301 c > T, P. (Arg 101 Trp) in exon 3 of the IL-10RA gene, which is a missense mutation and results in the conversion of the 101st amino acid from Arg to Trp. The other occurred (c. 537G > A, P. (Thr $179=$ ) in exon 4 of IL-10 RA gene, which is a synonymous mutation and can affect mRNA splicing. Moreover, her father carried a heterozygous mutation $(\mathrm{c} .301 \mathrm{c}>\mathrm{T}$, P. (Arg 101 Trp) in exon 3 of IL-10RA gene; and her mother carried a heterozygous mutation (c.537G > A, P. (Thr $179=$ ) in exon 4 of the IL-10RA gene. In other words, the patient had two mutations in the IL-10RA gene, one from her father and one from her mother. The results suggest that neonatal $C D$ is a disease of hereditary IL-10RA gene deficiency, with an obvious genetic background. In order to exclude the effect of diet on neonatal $\mathrm{CD}$, we paid attention to the feeding situation of the patient. The patient had been breast-fed from birth to hospitalization and did not eat any other dairy products. As a result, we can rule out the effect of environmental factors leading to neonatal CD in our patient. This study is of great significance to the classification of VIBD pathogenic factors and for the expanding the gene mutation spectrum of CD.

The IL-10R gene includes IL-10RA and IL-10RB. In order to improve the understanding of $\mathrm{CD} / \mathrm{IBD}$ caused by IL-10R gene mutations among neonatal pediatricians, we analyzed the characteristics of neonatal CD/IBD caused by IL-10R gene mutations. The results showed that a mutation in the IL-10RA gene (91.67\%) is more common than that in the IL-10RB gene (8.33\%). Compound heterozygous mutations (69.56\%) are also common; with the C. $301 \mathrm{C}>\mathrm{T}$ mutation being the first and C. $537 \mathrm{G}>\mathrm{A}(41.67 \%)$ being the second. Regarding clinical manifestation, diarrhea of unknown cause accounts for $82.26 \%$, mucous stools, bloody purulent stools, or bloody stools accounts for $69.57 \%$, and fever accounts for $65.52 \%$. With these early signs, doctors can easily consider neonatal CD/IBD; however, other symptoms or signs are often ignored by doctors, especially oral ulcers and erythra. If the patient only presents with oral ulcers, rash, and perianal disease, without diarrhea and stool abnormalities, most doctors would not consider neonatal
CD. We believe that this is the main reason for misdiagnosis and missed diagnosis of neonatal CD. Previous literature reports that the accuracy rate of fever, bleeding, and diarrhea in CD patients is $84.62 \%$ [32]. Clinically, oral lesions are rare in $\mathrm{CD}$ patients [33], accounting for only $4.8 \%$ of the patients [34]. Our patient' first symptom was oral ulcers before beginning to develop a rash, fever, perianal mass, and perianal pyoderma; she did not have any symptoms of diarrhea or abnormal stools after admission. As a result, we did not perform endoscopy or intestinal. We instead suspected that the patient had an autoimmune disease, and NGS was performed to obtain a clear diagnosis. It is probable that our patient did not present with diarrhea and abnormal stools because CD was early onset; therefore, we can understand the particularity of this case. Our case suggests that newborns admitted to hospital with oral ulcers, fever, rashes, and perianal lesions should be closely monitored and genetically screened for neonatal $\mathrm{CD}$, even if the patient has no symptoms of diarrhea and abnormal stools. This is important to make a clear diagnosis in the early stages of $\mathrm{CD}$ in newborns.

Patients with IL-10 or IL-10R gene deficiency often present with hematopoietic cell defects and immune regulation disorder, which can be dangerous for the patient. Thus far, there is no effective treatment for neonatal CD. According to the known of pathogenesis and previous literature reports, in addition to the use of conventional drugs and surgical intervention, immunosuppressive methods can be tried, such as anti-tumor necrosis factor $[35,36]$, anti-IL-12/23 [37, 38], IL- $1 \beta$ or hematopoietic stem cell transplantation, or gene therapy $[39,40]$. According to the present literature and our research, neonatal CD is most often caused by a IL-10RA gene mutation. However, further study is required regarding the prevalence of genetic variations in different ethnicities and regions.

In conclusion, neonatal $\mathrm{CD}$ is very rare. Mutations in IL-10RA, resulting in gene deficiency, is the main factor for neonatal $\mathrm{CD}$. The correlation between genotype and clinical manifestations requires further investigation.

\section{Abbreviations \\ CD: Crohn's disease; IL-10RA: Interleukin-10 receptor A}

\section{Acknowledgements}

Not applicable.

\section{Authors' contributions}

All authors participated in the design of the case report and coordination, and helped to draft the manuscript. All authors read and approved the final manuscript.

\section{Funding}

The authors received no financial support for the research, authorship, and/ or publication of this article. 


\section{Availability of data and materials}

The datasets generated during and analyzed during the current study are available from the corresponding author on reasonable request.

\section{Ethics approval and consent to participate}

This study was approved by the Medical Ethics Committee of Handan Maternal and Child Health Care Hospital of Hebei province. Written informed consent was provided by patient's parents.

\section{Consent for publication}

Written informed consent was provided by patient's parents.

\section{Competing interests}

The authors declare that they have no competing interests.

\section{Author details}

${ }^{1}$ Department of Neonatology, Handan Maternal and Child Health Care Hospital, No. 6, Li Ming Street, Peace Road, Handan City 056001, Hebei Province, China. ${ }^{2}$ Department of Neonatal Pathology, Handan Maternal and Child Health Care Hospital, No. 6, Li Ming Street, Peace Road, Handan City 056001, Hebei Province, China. ${ }^{3}$ Department of Neural Development and Neural Pathology, Hebei University of Engineering School of Medicine, Handan 056029, Hebei Province, China.

\section{Received: 10 August 2019 Accepted: 17 December 2019} Published online: 26 December 2019

\section{References}

1. Cottone M, Cipolla C, Orlando A, et al. Epidemiology of Crohn's disease in Sicily: a hospital incidence study from 1987-1989."the Sicilian study group of inflammatory bowel disease". Eur J Epidemiol. 1991;7:636-40.

2. Economou M, Pappas G. New Global Map of Crohn's Disease: Genetic, environmental,and socioeconomic correlations. Inflamm Bowel Dis. 2008; 14:709-20.

3. Ng SC, Tang W, Ching JY, et al. Incidence and phenotype of inflammatory bowel disease based on results from the Asia-pacific Crohn's and colitis epidemiology study. Gastroenterology. 2013;145:158-65 e2.

4. Li H, Durbin R. Fast and accurate long-read alignment with burrowswheeler transform. Bioinformatics. 2010;26:589-95.

5. Zhang L, Zhang J, Yang J, et al. A toolkit for prioritizing SNVs and indels from next-generation sequencing data. Bioinformatics. 2013;29:124-5.

6. Wang FP, Ma X, Zhu D, et al. Interleukin-10 receptor gene mutations induced very early onset inflammatory bowel disease in 6 infants. Chin J Pediatr. 2018:56:753-8.

7. Lu D, Xu Y, Chen Y, et al. Interleukin-10 recepotor mutation in children with neonatal onset inflammatory bowel disease: genetic diagnosis and pathogenesis. Zhonghua Er Ke Za Zhi. 2015;53:348-54.

8. Jiang $Y, C$ Chen $\mathrm{DH}$, Liu LL, et al. A study on the interleukin-10 receptor gene mutation and neonatal very early onset inflammatory bowel disease in 5 infants. Chin J Neonatol. 2017;32:105.

9. Shim JO, Hwang S, Yang HR, et al. Interleukin-10 receptor mutations in children with neonatalonset Crohn's disease and intractable ulcerating enterocolitis. Eur J Gastroenterol Hepatol. 2013;25:1235-40.

10. Fang $Y H$, Luo $Y Y, Y u$ JD, et al. Phenotypic and genotypic characterization of inflammatory bowel disease in children under six years of age in China. World J Gastroenterol. 2018;24:1035-45.

11. Yanagi T, Mizuchi T, Takaki Y, et al. Novel exonic mutation inducing aberrant splicing in the IL-10RA gene and resulting in ingfantile-onset inflammatory bowel disease: a case report. BMC Gastroenterol. 2016;16:10. https://doi.org/ 10.1186/s12876-016-0424-5.

12. Kotlarz D, Beier R, Murugan D, et al. Loss of interleukin-10 signaling and infantile inflammatory bowel disease: implications for diagnosis and therapy. Gastroenterology. 2012;143:347-55.

13. Oh SH, Baek J, Liany $H$, et al. A synonymous variant in IL-10RA affects RNA splicing in paediatric patients with refractory inflammatory bowel disease. J Clin Immunol. 2016;36:747-9.

14. Wang $X Q$, Zhang $Y, X u C D$, et al. Inflammatory bowel disease in Chinese chidren: a multicenter analysis over a decade from Shanghai. Inflamm Bowel Dis. 2013;19:423-8.

15. Kelsen JR, Baldassano RN. The role of monogenic disease in children with very early onset inflammatory bowel disease. Curr Opin Pediatr. 2017;29:566-71.
16. Abraham C, Cho JH. Inflammatory bowel disease. N Engl J Med. 2009;361: 2066-78.

17. Ekbom A, Daszak P, Kraaz W, et al. Crohn's disease after in-utero measles virus exposure. Lancet. 1996:348:515-7.

18. Nielsen LL, Nielsen NM, Melbye M, et al. Exposure to measles in utero and Crohn's disease: Danish register study. BMJ. 1998;316:196-7.

19. Haslam N, Mayberry JF, Hawthorne AB, et al. Measles, month of birth, and Crohn's disease. Gut. 2000;47:801-3.

20. Sørensen HT, Pedersen L, Nøgård B, et al. Does month of birth affect risk of Crohn's disease in childhood and adolescence. BMJ. 2001;323:907.

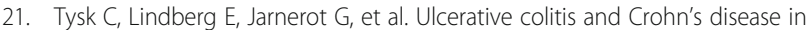
an unselected population of monozygotic and dizygotic twins: a study of heritability and the influence of smoking. Gut. 1988;29:990-6.

22. Fang YH, Luo YY, Yu JD, et al. Phenotypic and genotypic characterization of inflammatory bowel disease in children under sis years of age in China. World J Gastroenterol. 2018;24:1035-45 PMID: 29531467.

23. Li HY, Ju R. A case report of Crohn's disease in neonates. Chin J Neonatol. 2017;32:305

24. Uhlig HH, Schwerd T, Koletzko S, et al. The diagnostic approach to monogenic very early onset inflammatory bowel disease. Gastroenterology. 2014;147:990-1007 es.

25. Glocker EO, Kotlarz D, Boztug K, et al. Inflammatory bowel disease and mutations affecting the interleukin-10 receptor. N Engl J Med. 2009;361: 2033-45.

26. de Ridder L, Weersma RK, Dijkstra G, et al. Genetic susceptibility has a more important role in pediatric-onset Crohn's disease than in adult-onset Crohn's disease. Inflamm Bowel Dis. 2007;13:1083-92.

27. Moran CJ, Walters TD, Guo CH, et al. IL-10R polymorphisms are associated with very-early-onset ulcerative colitis. Inflamm Bowel Dis. 2013;19:115-23.

28. Xiao Y, Wang XQ, Yu Y, et al. Comprehensive mutation screening for 10 genes in Chinese patients suffering very early onset inflammatory bowel disease. World J Gastroenterol. 2016;22:5578-88.

29. Holm AM, Aukrust P, Aandahl EM, et al. Impaired secretion of IL-10 by T cells from patients with common variable immunodeficiency--involvement of protein kinase A type I. J Immunol. 2003:170:5772-7.

30. Kelsen JR, Dawany N, Moran CJ, et al. Exome sequencing analysis reveals variants in primary immunodeficiency genes in patients with very early onset inflammatory bowel disease. Gastroenterology. 2015;149:1415-24.

31. Huang Z, Peng K, Li X, et al. Mutations in interleukin-10 receptor and clinical phenotypes in patient with very early onset infammatory bowel disease:a Chinese VEO- IBD collaboration group survey. Inflamm Bowel Dis. 2017;23: 578-90.

32. Amarapurkar DN, Patel ND, Rane PS. Diagnosis of Crohn's disease in India where tuberculosis is widely prevalent. World J Gastroenterol. 2008;14:741-6.

33. Bokemeyer A, Tentrop N, Barth PJ, et al. Successful treatment of oral Crohn's disease by anti-TNF alpha dose escalation- a case report. BNC Gastroenterol. 2018;18:88.

34. Hopkins DJ, Horan E, Burton IL, Burton IL, et al. Ocular disorders in a series of 332 patients with Crohn's disease. Br J Ophthal. 1974;58:732-7.

35. O'Neill ID, Scully C. Biologics in oral medicine: oral Crohn's disease and orofacial granulomatosis. Oral Dis. 2012;18:633-8.

36. Vavricka SR, Gubler M, Gantenbein C, et al. Anti-TNF treatment for Extraintestinal manifestations of inflammatory bowel disease in the Swiss IBD cohort study. Inflamm Bowel Dis. 2017;23:1174-81.

37. Bernstein CN. Treatment of IBD: where we are and where we are going. Am J Gastroenterol. 2015;110:114-26.

38. Lazzerini M, Villanacci V, Pellegrin MC, et al. Endoscopic and Histologic Healing in Children With Inflammatory Bowel Diseases Treated With Thalidomide. Clin Gastroenterol Hepatol. 2017;15:1382.e1-9.e1.

39. Engelhardt KR, Shah N, Faizura-Yeop I, et al. Clinical outcome in IL-10- and IL-10 receptor-deficient patients with or without hematopoietic stem cell transplantation. J Allergy Clin Immunol. 2013;131:825-30.

40. Uhlig $\mathrm{HH}$. Monogenic diseaaes associated with intestinal inflammation: implication for the understanding of inflammatory bowel disease. Gut. 2013; 62:1795-805.

\section{Publisher's Note}

Springer Nature remains neutral with regard to jurisdictional claims in published maps and institutional affiliations. 